Draft VERsion August 24, 2021

Preprint typeset using LTEX style emulateapj v. 12/16/11

\title{
SUZAKU OBSERVATIONS OF THE TYPE 2 QSO IN THE CENTRAL GALAXY OF THE PHOENIX CLUSTER
}

\author{
Shutaro Ueda $^{1}$, Kiyoshi Hayashida ${ }^{1}$, NaOhisa Anabuki ${ }^{1}$, Hiroshi NaKajima ${ }^{1}$, Katsuji Koyama ${ }^{1,2}$, And Hiroshi Tsunemi ${ }^{1}$
}

Draft version August 24, 2021

\begin{abstract}
We report the Suzaku/XIS \& HXD and Chandra/ACIS-I results on the X-ray spectra of the Phoenix cluster at the redshift $z=0.596$. The spectrum of the intracluster medium (ICM) is well-reproduced with the emissions from a low temperature $(\sim 3.0 \mathrm{keV}$ and $\sim 0.76$ solar $)$ and a high temperature $(\sim 11 \mathrm{keV}$ and $\sim 0.33 \mathrm{solar})$ plasmas; the former is localized at the cluster core, while the latter distributes over the cluster. In addition to these ICM emissions, a strongly absorbed power-law component is found, which is due to an active galactic nucleus (AGN) in the cluster center. The absorption column density and unobscured luminosity of the AGN are $\sim 3.2 \times 10^{23} \mathrm{~cm}^{-2}$ and $\sim 4.7 \times 10^{45} \mathrm{ergs} \mathrm{s}^{-1}(2-10 \mathrm{keV})$, respectively. Furthermore, a neutral iron (Fe I) $\mathrm{K}$-shell line is discovered for the first time with the equivalent width (EW) of $\sim 150 \mathrm{eV}$ at the rest frame. The column density and the EW of the Fe I line are exceptionally large for such a high luminosity AGN, and hence the AGN is classified as a type 2 quasi-stellar object (QSO). We speculate that the significant fraction of the ICM cooled gas would be consumed to maintain the torus and to activate the type 2 QSO. The Phoenix cluster has a massive starburst in the central galaxy, indicating suppression in the cooling flow is less effective. This may be because the onset of the latest AGN feedback has occurred recently and it has not yet been effective. Alternatively, the AGN feedback is predominantly in radiative-mode not in kinetic-mode and the torus may work as a shield to reduce its effect.

Keywords: galaxies: clusters: individual: (Phoenix cluster, SPT-CLJ2344-4243) - X-rays: galaxies: clusters — galaxies: active — quasars: general
\end{abstract}

\section{INTRODUCTION}

Brightest cluster galaxies (BCGs) are giant elliptical galaxies located at the centers of the clusters. BCGs host supermassive black holes (SMBHs) at their nuclei as for other massive galaxies. BCGs and SMBHs in them are located at the highest end of the well known $M_{\mathrm{BH}}$ and $\sigma$ relation, where $M_{\mathrm{BH}}$ is the mass of a SMBH and $\sigma$ is the velocity dispersion of the bulge of a galaxy (e.g. Ferrarese \& Merritt 2000; Gebhardt et al. 2000; Tremaine et al. 2002; Sadoun \& Colin 2012; Salviander \& Shields 2013; McConnell \& Ma 2013). However, a large scatter or deviation from the $M_{\mathrm{BH}}$ and $\sigma$ relation extrapolated from the non BCG sample is found for the SMBHs in BCGs. Observational evidence and numerical simulations indicate that highest mass SMBHs $(\sim$ $10^{10} M_{\odot}$ ) and their host BCGs undergo some unique history of merging and/or accretion processes (e.g. Hopkins et al. 2007; Gültekin et al. 2009; McConnell et al. 2011, 2012; Graham \& Scott 2013; Volonteri \& Ciotti |2013).

The number density of distant quasi-stellar objects (QSOs) is larger than that of nearby QSOs (Richards et al. 2006), while McConnell et al. (2011) found that the number density of nearby BCGs is consistent with that of SMBHs in the highest mass limit $\left(10^{9}-10^{10} M_{\odot}\right)$, which are predicted from the $M_{\mathrm{BH}}-L$ relation (e.g. Ferrarese \& Merritt 2000; McLure \& Dunlop 2002; Marconi \& Hunt 2003) and the luminosity function of nearby galaxies. McConnell et al. (2011) suggests that local BCGs host the remnants of highly luminous QSOs. Some numerical simulations indicate that lumi-

\footnotetext{
shutaro@ess.sci.osaka-u.ac.jp

${ }^{1}$ Department of Earth and Space Science, Graduate School of Science, Osaka University, 1-1, Machikaneyama, Toyonaka, Osaka, 560 0043, Japan

2 Department of Physics, Graduate School of Science, Kyoto University, Kita-Shirakawa, Sakyo-ku, Kyoto, 606-8502, Japan
}

nous QSOs in the high-redshift end up as QSOs in the massive central galaxies (i.e. BCGs) of rich clusters at the local Universe (e.g. Springel et al. 2005, 2006; Li et al. 2007; Angulo et al. 2012). Observationally, De Lucia \& Blaizot (2007) shows that high-redshift BCGs belong to the same populations of local BCGs in their massive end, while Husband et al. (2013) indicates that the luminous QSOs at $z \sim 5$ likely represent an early stage in building-up massive low-redshift clusters. QSOs would be very active in the early Universe, possibly at $z=2-4.5$, but are dormant at present (e.g. Richards et al. 2006; Vestergaard et al. 2008). For example, the prominent radio-jet galaxy M87 in the Virgo cluster hosts a SMBH of $6.3 \times 10^{9} M_{\odot}$ (McConnell et al. 2011), but the present luminosity is many orders of magnitude lower than the Eddington limit (Di Matteo et al. 2003), much lower than those of typical active galactic nuclei (AGNs).

Detailed study of the active phase of the SMBHs in BCGs is difficult for the sources at $z>1$, but there are exceptional cases in which QSOs are found in the BCGs of clusters at lower redshift. In such sources, SMBHs are surely growing by gas accretion (e.g. Salpeter 1964; Hopkins et al. 2005), while strong radiation and/or jets from the SMBHs might affect the intracluster medium (ICM) in clusters (e.g. Fabian 2012). Therefore, those sources are of extreme importance for the study of the feeding and feedback processes in SMBHs, BCGs, and clusters.

Several such candidates include E1821+643 (Kii et al. 1991; Yamashita et al. 1997; Jiménez-Bailón et al. 2007; Russell et al. 2010), 3C 186 (Siemiginowska et al. 2010), PKS 1229-021 (Russell et al. 2012), IRAS 09104+4109 (Iwasawa et al. 2001; Vignali et al. 2011; O'Sullivan et al. 2012), IRAS F15307+3252 (Iwasawa et al. 2005), and the Phoenix cluster (McDonald et al.|2012, 2013). Among them, we select the Phoenix cluster at the redshift $z=0.596$, hosting 
a massive BCG with very luminous SMBH.

An X-ray emission from the Phoenix cluster is firstly reported as 1RXS J234444.1-424319 in the ROSAT Bright Source Catalog (Voges et al. 1999). This source is classified to be a Seyfert 2 in the Quasar and AGN Cata$\log 10$ th Edition by Véron-Cetty \& Véron (2001). The Two Micron All Sky Survey (2MASS) found an extended source 2MASX J23444387-423124 (Skrutskie et al. 2006), while the Palermo Swift/BAT hard X-ray catalog source, 2PSBC J2344.8-4245 is identified as 2MASX J23444387423124 (Cusumano et al. 2010). The $14-150 \mathrm{keV}$ band luminosity is extremely high as $1.4 \pm 0.9 \times 10^{46} \mathrm{ergs} \mathrm{s}^{-1}$.

First identification of this source as a cluster was, however, made with the South Pole Telescope (SPT) via the Sunyaev-Zel'dovich effect, and named as SPT-CLJ2344-4243 (Williamson et al. 2011). With radio, infrared, optical, ultraviolet, and X-ray observations of this source, McDonald et al. (2012) reported that the X-ray luminosity within $r_{500}$ and the total mass within $r_{200}$ of this cluster are $8.2 \times 10^{45} \mathrm{ergs} \mathrm{s}^{-1}$ in $2-10 \mathrm{keV}$ and $2.5 \times 10^{15} M_{\odot}$, respectively. Both are exceptionally large compared to the other known clusters.

Using Hubble Space Telescope and Chandra data (the same data as McDonald et al. 2012), McDonald et al. (2013) estimated that the SFR and cooling rate are $798 \pm 42 M_{\odot} \mathrm{yr}^{-1}$ and $2700 \pm 700 M_{\odot} \mathrm{yr}^{-1}$ (also from White et al. 1997), respectively. Thus, the SFR is $30 \pm 8 \%$ of the cooling rate, which is one of the highest among typical cool-core clusters in the local Universe. McDonald et al. (2012) also reported the central AGN has the luminosity of $\sim 3 \times 10^{45} \mathrm{ergs} \mathrm{s}^{-1}(2-10 \mathrm{keV})$ with a large absorption of $\sim 3.9 \times 10^{23} \mathrm{~cm}^{-2}$.

Apart from these general features of the Phoenix cluster, no detailed X-ray spectroscopy especially for the central AGN, and hence no accurate physical parameters of accretion and obscuration from X-ray observation (e.g. Mushotzky et al. 1993), has been examined so far, probably due to the limited statistics in the previous observations. Furthermore, the Swift/BAT hard X-ray, a key band to evaluate the intrinsic luminosity of the AGN, would be contaminated by the thermal plasma in the cluster depending on the plasma temperature. We therefore examined the high quality data of the deep Suzaku observation. To separately examine the central AGN component from the thermal emission of the ICM, we also employed the Chandra archival data.

In this paper, we adopt the abundance table of Anders \& Grevesse (1989), the Hubble constant of $H_{0}=$ $70 \mathrm{~km} \mathrm{~s}^{-1} \mathrm{Mpc}^{-1}, \Omega_{\mathrm{M}}=0.27$, and $\Omega_{\Lambda}=0.73$. One arcsec corresponds to $6.7 \mathrm{kpc}$ at the redshift $z=0.596$ for this cluster. Unless otherwise specified, all errors represent at $90 \%$ confidence level (90\% CL).

\section{OBSERVATIONS AND DATA REDUCTIONS}

A Suzaku (Mitsuda et al. 2007) observation of the Phoenix cluster was performed on Nov. 15th-16th 2010 (ObsID:70549010, PI: W. Baumgartner). Data process and reduction were done with the HEASOFT version 6.12 (e.g. xispi, xselect). We first reprocessed the unfiltered event files in the Suzaku data archive and the calibration data base (CALDB) released on Oct. 15th 2012. Other information was derived from the Suzakuteam 3 . After the standard data reduction, the exposure times were $62 \mathrm{ks}$ and $47 \mathrm{ks}$ for the X-ray Imaging Spectrometer (XIS: Koyama et al. 2007b)

\footnotetext{
${ }^{3}$ http://heasarc.gsfc.nasa.gov/docs/suzaku/analysis/abc/
}

and the Hard X-ray Detector (HXD: Takahashi et al. 2007; Kokubun et al. 2007), respectively.

The XIS (XIS0, XIS1, and XIS3) data were extracted from the circular region with a radius of $3^{\prime}$ centered on the BCG of the Phoenix cluster as shown in the left panel of Figure 1 The non X-ray background (NXB) of the XIS was estimated by using the database of night earth observations with xisnxbgen (Tawa et al. 2008). For the extracted data, we made the NXB-subtracted light curve of XIS0, 1, and 3 in the $0.4-10 \mathrm{keV}$ band from the $r<3^{\prime}$ region with the time bin size of $1024 \mathrm{~s}$. All the data points are within the range of $\pm 3 \sigma\left( \pm 0.10 \mathrm{cts} \mathrm{s}^{-1}\right)$ of the mean $\left(1.08 \mathrm{cts} \mathrm{s}^{-1}\right)$, and hence no significant variability (no anomaly) in the data is found, or no flickering event is included.

We also used the Chandra archival data of this source (ObsID:13401, PI: G. Garmire), which are the same data used by McDonald et al. (2012). The observation was carried out on Sep.19th 2011 for an exposure time of $12 \mathrm{ks}$ with the Advanced CCD Imaging Spectrometer (ACIS: Garmire et al. 2003). We reprocessed and reduced the level 2 Chandra/ACIS-I event data by using the Chandra Interactive Analysis of Observations (CIAO) version of 4.4.1 and the CALDB version 4.5.3.

\section{SPECTRAL ANALYSES AND RESULTS}

In the analysis of the Suzaku spectra, the NXB is subtracted by using the database (for the XIS) or model (for the HXD), respectively. The cosmic $\mathrm{X}$-ray background $(\mathrm{CXB})$ is given by the cut-off power-law (Boldt 1987). The Milky Way halo (MWH) and the local hot bubble (LHB) are described by the APEC models (Smith et al. 2001) of $0.23 \mathrm{keV}$ and $0.07 \mathrm{keV}$, respectively (Ueda et al. 2013). In the spectral fitting, these $\mathrm{X}$-ray background models with the Galactic absorption (wabs model, Morrison \& McCammon 1983) of the column density $\left(N_{\mathrm{H}}\right) 1.52 \times 10^{20} \mathrm{~cm}^{-2}$ (Kalberla et al. 2005) are added to the source model.

The Redistribution Matrix Files (RMFs) and Ancillary Response Files (ARFs) of the XIS are generated with xisrmfgen and xissimarfgen (Ishisaki et al. 2007), respectively.

The HXD consists of two detectors, PIN and GSO, but we used only the PIN data. The RMF (ae_hxd_pinhxnome9_20100731.rsp) and the $\mathrm{NXB}$ are released by the $\mathrm{HXD}$ calibration team. The cross-calibration error in the effective areas of different detectors (XIS and HXD) is compensated by multiplying 1.181 to the normalization of the HXD (Suzaku Memd). The normalization of the CXB for the HXD is fixed at the values supplied by the HXD team.

For the Chandra/ACIS-I spectra, on the other hand, we subtract the NXB plus the X-ray backgrounds which were made from the region beyond $7^{\prime}$ from the center in the same field of the Phoenix cluster. The RMFs and the ARFs are generated by using specextract.

\subsection{Suzaku/XIS \& HXD spectra in the $r<3^{\prime}$ region}

We show the Suzaku/XIS image of the Phoenix cluster field in the left panel of Figure 1 The spectra are extracted from the $r<3^{\prime}$ region (the solid circle in the left panel of Figure 1); those from XIS0 and XIS3 are restricted to the $0.4-10 \mathrm{keV}$ range, while the XIS1 spectrum is restricted to $0.4-7 \mathrm{keV}$. In

\footnotetext{
${ }^{4} \mathrm{ftp} / / /$ legacy.gsfc.nasa.gov/suzaku/doc/xrt/suzakumemo-2008-06.pdf
} 

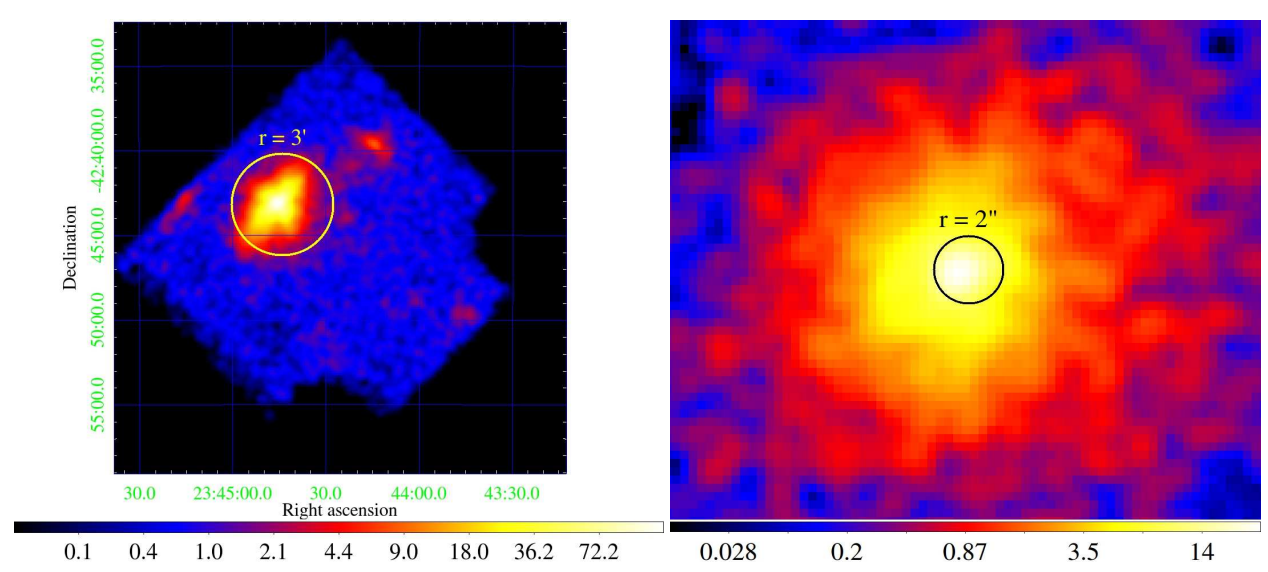

Figure 1. X-ray images of the Phoenix cluster in the $0.4-10 \mathrm{keV}$ band. The background is not subtracted and the vignetting effect is not corrected. The unit of color bar is counts pixel ${ }^{-1}$. Left panel: X-ray image of Suzaku/XIS3. The yellow circle shows the region of $r<3^{\prime}$. Right panel: The Chandra/ACIS-I image of central region of the Phoenix cluster. The image is smoothed with a 2-dimensional Gaussian of $\sigma=3$ pixels. The black line is the $r=2^{\prime \prime}$ circle.

the rest frame of $z=0.596,3^{\prime}$ corresponds to $1.2 \mathrm{Mpc}$, and hence the $r<3^{\prime}$ circle includes the major fraction of the cluster emission.

Since the Suzaku/HXD is a non-imaging instrument, the spectral data are from the PIN field of $34^{\prime} \times 34^{\prime}$. We employ the energy range of $16-40 \mathrm{keV}$. The HXD count rate from the source (the NXB and the CXB subtracted) is $1.5 \pm$ $0.2 \times 10^{-2} \mathrm{cts} \mathrm{s}^{-1}$ in $16-40 \mathrm{keV}$, which corresponds to $7.3 \%$ of the NXB, significantly larger than the systematic error in the NXB of $2.1-2.7 \%(1 \sigma)$ (Takahashi et al. 2010).

In addition to the $\mathrm{X}$-ray background model (see section 3 ), we apply a single temperature (1T) thin thermal plasma model (APEC) as the spectrum of the Phoenix cluster. Then, we obtain the gas temperature, abundances, and redshift of the Phoenix cluster, as $16.7_{-0.8}^{+0.9} \mathrm{keV}, 0.81 \pm 0.09$ solar, and $0.656_{-0.005}^{+0.007}$, respectively. This 1T model, however, shows the over-all spectral shape with the concave residual, in excess at the low and high energy bands (see the top left panel of Figure 2). Furthermore, the best-fit redshift $z=0.656$ is inconsistent with the optical observations (McDonald et al. 2012). We also find a line-like residual at $4.3 \mathrm{keV}$, which corresponds to $\sim 7 \mathrm{keV}$ after correcting the redshift of 0.656 . Thus, the residual may be either due to misidentification of the K-shell lines, and hence gave a larger plasma temperature as $16.7 \mathrm{keV}$, or due to an additional iron $\mathrm{K}$-shell line.

The excess at the low energy band may indicate the presence of another thin thermal plasma component with a low temperature. Two-temperature structure is already suggested in the 1T fitting of the spatially resolved Chandra/ACIS-I spectra (McDonald et al. 2012), which shows a low temperature in the inner region of $r \lesssim 100 \mathrm{kpc}$, and high temperature in the outer region. The excess at the high energy band suggests the presence of a power-law component at the cluster center.

\subsection{Chandra/ACIS-I spectra from the inner and outer regions}

We show the Chandra/ACIS-I image to highlight the core and surrounding envelope of the Phoenix cluster in the right panel of Figure1 Then, we extract the Chandra/ACIS-I spectra from the inner region of $r<2^{\prime \prime}$, and the outer region of $2^{\prime \prime}<r<3^{\prime}$ annulus.

To examine the high temperature plasma in the outer region, we fit the spectrum with an APEC model fixing the redshift to $z=0.596$. This model gives a nice fit with $\chi^{2} /$ d.o.f. $=89 / 122$, as is shown in the top right panel of Figure 2 The best-fit temperature and abundance are constrained to be $10.9_{-1.1}^{+1.8} \mathrm{keV}$ and $0.33_{-0.16}^{+0.18}$ solar, respectively. They are roughly consistent with those obtained by McDonald et al. (2012).

We then examine the spectrum from the inner region of $r<2^{\prime \prime}$. As is shown in the bottom left panel of Figure 2 this spectrum has a local minimum at about $2 \mathrm{keV}$, which indicates the presence of a soft component plus highly absorbed hard component. The former would be a low temperature plasma, while the latter is likely an AGN. We therefore fit the spectrum with an APEC model (for low temperature plasma) plus an absorbed power-law (absPL) continuum (for AGN). This model is accepted with $\chi^{2} /$ d.o.f. $=45 / 42$. We thus conclude that the X-rays from the inner region of the Phoenix cluster consist of a low temperature component and an AGN power-law component. However, the physical parameters are only loosely constrained. The best-fit photon index and absorption column density for the power-law component are $\Gamma=0.71_{-0.66}^{+0.82}$ and $N_{\mathrm{H}}=1.9_{-1.2}^{+2.4} \times 10^{23} \mathrm{~cm}^{-2}$, respectively, while the temperature and abundance for the low temperature plasma are $4.89_{-1.80}^{+6.07} \mathrm{keV}$ and $1.48_{-1.13}^{+2.26}$ solar, respectively. Table 1 summarizes the best-fit values of these fittings.

\subsection{Suzaku/XIS fit in the $3.5-5.0 \mathrm{keV}$}

In the 1T model fit for the Suzaku spectra (subsection 3.1), we also found a significant line-like residual at the energy at $\sim 4 \mathrm{keV}$. Converting it to the rest frame, the line energy corresponds to either the K-shell lines from neutral iron (Fe I), He-like Fe XXV or H-like Fe XXVI. We therefore zoom-up the XIS spectrum in the $3.5-5.0 \mathrm{keV}$ range, and fit with a power-law continuum plus three Gaussian lines, in which the line energies are fixed to those of the K-shell transition from Fe I, Fe XXV, and Fe XXVI at the redshift $z=0.596$. The spectrum and the best-fit results are shown in the bottom right panel of Figure 2, The Fe I line is detected at 3.5 $\sigma$ level. The equivalent width (EW) defined to the summed continuum of the thermal (i.e. the ICM emissions) and non-thermal (i.e. the central AGN emission) components is $23_{-11}^{+10} \mathrm{eV}$ at the observer frame. Since a thin thermal plasma cannot emit the Fe I line, the most likely origin is an AGN in the BCG.

As we suggest in subsection 3.1 , we find that the $1 \mathrm{~T}$ model 
fit of the wide band spectra misidentified the Fe XXV line to that of Fe XXVI and Fe I to Fe XXV, and hence misled to a larger redshift of 0.656 and higher temperature of $k T \sim 17 \mathrm{keV}$.

\subsection{Simultaneous fit for the Suzaku/XIS \& HXD and Chandra/ACIS-I spectra in the $r<3^{\prime}$ region}

We finally carry out the simultaneous fit for the X-ray spectra extracted in the same $r<3^{\prime}$ region from the Suzaku/XIS \& HXD and Chandra/ACIS-I. The model includes all the components found in the previous subsections. Schematically the spectral model is given as wabs $\times\left(\mathrm{APEC}_{\text {low }}+\right.$ $\mathrm{APEC}_{\text {high }}+$ zwabs $\times$ power-law + zgauss $)$. We fix the temperature and abundance of $\mathrm{APEC}_{\text {high }}$ component as those obtained in the spectral fit with the Chandra/ACIS-I spectrum of the $2^{\prime \prime}<r<3^{\prime}$ region. The line center energy was fixed at $6.40 \mathrm{keV}$ (i.e. the Fe I line) at the rest frame. Since other parameters determined in the previous subsections are not well constrained, we treat them as free parameters. This model $(2 \mathrm{~T}+\mathrm{absPL}+\mathrm{Fe} \mathrm{I})$ nicely reproduces the over-all spectra with $\chi^{2} /$ d.o.f. $=790 / 732$. The best-fit model and the data residual are shown in Figure 3, while the best-fit parameters are summarized in Table 1 All the best-fit parameters are consistent with those determined by the individual spectral fit given in subsections 3.2 and 3.3 but are more accurately determined. For comparisons with the previous results, we calculate the $10-50 \mathrm{keV}$ and $14-150 \mathrm{keV}$ band luminosities for the power-law component as $9.4_{-0.2}^{+0.1} \times 10^{45} \mathrm{ergs} \mathrm{s}^{-1}$ and $2.1_{-0.8}^{+0.7} \times 10^{46} \mathrm{ergs} \mathrm{s}^{-1}$, respectively. The EW of the Fe I line defined to the continuum emission of the central AGN (i.e. the absorbed power-law component) is $149_{-58}^{+139} \mathrm{eV}$ at the rest frame.

\section{DISCUSSION}

With the simultaneous fit of the Suzaku/XIS \& HXD and the Chandra/ACIS-I data, we have discovered that the X-ray spectrum of the Phoenix cluster can be approximated by three components; two thin thermal plasma components with different temperatures and spatial distributions, and a power-law component in the cluster center. We also determine the redshift of the Phoenix cluster as $z=0.599_{-0.006}^{+0.004}$ by the X-ray spectra alone. This value is consistent with the mean value $(z=0.596 \pm 0.002)$ of 26 member galaxies (McDonald et al. 2012). In the following subsections, we separately discuss on the thermal emission from the ICM and the power-law component of the AGN. We then discuss possible interactions between these components through the viewpoint of the feeding and feedback processes in cluster, BCG, and SMBH. We refer all the physical parameters at the rest frame of $z=0.596$ unless otherwise specified.

\subsection{Thermal emission from the ICM}

Although the ICM may consist of multi-temperature plasmas in pressure equilibrium, it can be nicely approximated by two temperature plasma; the low temperature plasma of $2.95_{-0.48}^{+0.53} \mathrm{keV}$ and the high temperature plasma of $10.9_{-1.1}^{+1.8} \mathrm{keV}$. The X-ray luminosity in the $2-10 \mathrm{keV}$ band within $1.2 \mathrm{Mpc}$ are $L_{\mathrm{X}, \text { low }}=1.0_{-0.5}^{+0.4} \times 10^{45} \mathrm{ergs} \mathrm{s}^{-1}$ and $L_{\mathrm{X}, \mathrm{high}}=$ $7.0_{-0.6}^{+0.7} \times 10^{45} \mathrm{ergs} \mathrm{s}^{-1}$ for the low temperature and high temperature plasmas, respectively. The high temperature plasma is prevailing in the whole cluster with the abundances of $0.33_{-0.16}^{+0.18}$ solar, typical value for clusters. The low temperature plasma has a higher abundance of $0.76_{-0.31}^{+0.63}$ solar and is confined in the core of the cluster. Possibly in the cluster core, a large amount of metals is supplied from supernovae in the BCG. These features are commonly observed in nearby coolcore clusters with $\mathrm{cD}$ galaxies (i.e. BCG) at their centers (e.g. Matsushita 2011).

Following White et al. (1997), which is the same method as McDonald et al. (2013), we estimate the cooling rate to be $2290_{-770}^{+1260} M_{\odot} \mathrm{yr}^{-1}$. This value is consistent with that of McDonald et al. (2013).

\subsection{What is the origin of the Fe I line?}

As mentioned in section 3.3, we discover the Fe I line at the rest frame in the redshift of $z=0.596$. We discuss whether the Fe I line is due to the central AGN or to other possibilities.

One possibility is due to gain error of the Suzaku/XIS. The gain error of the Suzaku/XIS is $\sim 0.1 \%$ at $6 \mathrm{keV}(6 \mathrm{eV}$ for the Fe I line), which is estimated using several targets and Mn I K $\alpha$ lines from the calibration source of ${ }^{55} \mathrm{Fe}$ (e.g. Koyama et al. 2007a; Ota et al. 2007; Ozawa et al. 2009; Tamura et al. 2011). The energy difference between Fe I and Fe XXV at the observer's frame is $\sim 200 \mathrm{eV}$, which is significantly larger than the gain error of the Suzaku/XIS.

Second possibility is velocity-broadened (e.g. bulk motion and/or turbulence of the ICM) of Fe XXV line (6.7 keV at the rest frame), which may partly mimics as the Fe I line. We fitted the X-ray spectra (the lower right panel of Figure 2) with a broadened line of the Fe XXV line. Then, we obtain the line width of Fe XXV to be $\sim 188 \mathrm{eV}$ at the observer's frame, which corresponds to the velocity of $\sim 13000 \mathrm{~km} \mathrm{~s}^{-1}$ at the rest frame (Ota 2012). This velocity is too huge for the realistic motion of the ICM. Furthermore, McDonald et al. (2012) reported that the X-ray surface brightness of the Phoenix cluster shows a relaxed morphology of the ICM, suggesting bulk motion is not prominent.

Third possibility is a contamination of other point sources than the AGN in the BCG within $3^{\prime}$ radius. We evaluate such an amount by examining the $2-10 \mathrm{keV}$ intensity of the sources based on using the Chandra $11.9 \mathrm{ks}$ observation. The emission within $2^{\prime \prime}$ from the central AGN contains total 501 counts in $2-10 \mathrm{keV}$ band. Based on our fitting result shown in the lower left panel of Figure 2, we estimate that 361 counts is owing to the central AGN and the rest is primarily consists of the ICM thermal emission. On the other hand, there found six other point sources within $3^{\prime}$ radius around the central AGN. The most intense one has 8 counts in $2-10 \mathrm{keV}$ band, and sum of the counts from the six point sources is only 15 counts, which is $4 \%$ of that of the central AGN. Suppose that most of the six point sources are type I AGN with typical Fe I EW of $\sim 100 \mathrm{eV}$ (see e.g., the black triangles in the right panel of Figure 4), possible contribution for the Fe I EW of the central AGN is only $4 \mathrm{eV}$.

As discussed above, we can reject three possibilities for the origin of the $\mathrm{Fe} \mathrm{I}$ line. We hence regard the most natural idea is that the Fe I line comes from the central AGN in the BCG of the Phoenix cluster.

\subsection{Properties of the type $2 Q S O$ at the center}

We have discovered that the power-law component in the cluster center has a Fe I K-shell line with EW of $149_{-58}^{+139} \mathrm{eV}$. The power-law photon index, $\Gamma$, is determined for the first time as $1.54_{-0.24}^{+0.27}$. The absorption column density of $3.2_{-0.8}^{+0.9} \times$ $10^{23} \mathrm{~cm}^{-2}$ is consistent with, but is more accurate compared 

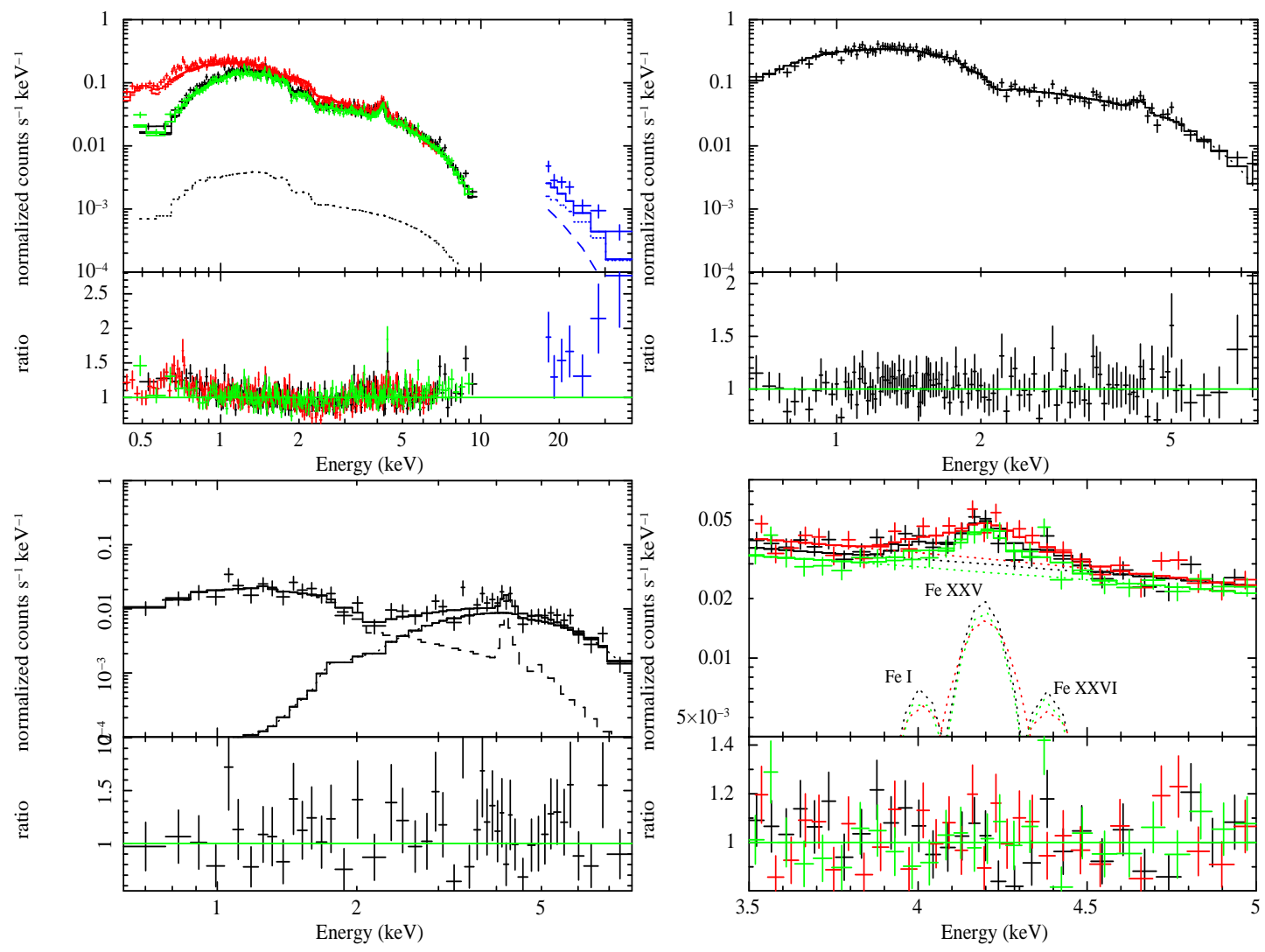

Figure 2. X-ray spectra of the Phoenix cluster. The ratios of the data to the model are plotted in the bottom panels. Top left panel: Suzaku/XIS0 (black), XIS1 (red), XIS3 (green), HXD (blue) spectra in the $r<3^{\prime}$ regions centered at the BCG fitted with a 1T model. The dashed lines show the best-fit model of the ICM for XIS0 and the HXD. The dotted lines represent the X-ray background model consisting of CXB, MWH, and LHB for XIS0 and HXD. Top right panel: Chandra/ACIS-I spectrum fitted with a 1T model. The region is $2^{\prime \prime}<r<3^{\prime}$. Bottom left panel: Chandra/ACIS-I spectrum in the core region $\left(r<2^{\prime \prime}\right)$ fitted with a $1 \mathrm{~T}$ model and an absorption power-law model. Bottom right panel: Suzaku/XIS spectra in 3.5-5.0 keV fitted with a power-law continuum and three Gaussian lines.

Table 1

Best-fit results of the spectral fitting with the Chandra/ACIS-I in $0.6-8 \mathrm{keV}$ and the Suzaku/XIS \& HXD and the Chandra/ACIS-I in $0.4-40 \mathrm{keV}$.

\begin{tabular}{|c|c|c|c|c|c|c|c|c|}
\hline \multirow{4}{*}{$\begin{array}{l}\text { Instruments } \\
\text { Chandra/ACIS-I }\end{array}$} & \multirow{4}{*}{$\begin{array}{l}\text { Region } \\
r<2^{\prime \prime}\end{array}$} & \multicolumn{2}{|c|}{$\mathrm{APEC}_{\text {low }}$} & \multicolumn{2}{|c|}{$\mathrm{APEC}_{\text {high }}$} & \multicolumn{2}{|c|}{ zwabs $\times$ power-law+zgauss } & \multirow{4}{*}{$\frac{\chi^{2} / \text { d.o.f. }}{45 / 42}$} \\
\hline & & $k T[\mathrm{keV}]$ & $4.89_{-1.80}^{+6.07}$ & & & $N_{\mathrm{H}}\left[\times 10^{23} \mathrm{~cm}^{-2}\right]$ & $1.9_{-1.2}^{+2.4}$ & \\
\hline & & $Z$ [solar] & $1.48_{-1.13}^{+2.80}$ & & & Photon index & $0.71_{-0.66}^{+0.22}$ & \\
\hline & & Redshift & 0.596 (fix) & & & EW of Fe I [eV] & $134_{-134}^{+207}$ & \\
\hline \multirow[t]{3}{*}{ Chandra/ACIS-I } & $2^{\prime \prime}<r<3^{\prime}$ & & & $k T[\mathrm{keV}]$ & $10.9_{-11}^{+1.8}$ & & & $89 / 122$ \\
\hline & & & & $Z$ [solar] & $0.33_{-0.16}^{+0.1}$ & & & \\
\hline & & & & Redshift & 0.596 (fix) & & & \\
\hline \multirow[t]{3}{*}{ Suzaku/XIS \& HXD and Chandra/ACIS-I } & $r<3^{\prime}$ & $k T[\mathrm{keV}]$ & $2.95_{-0.48}^{+0.53}$ & $k T[\mathrm{keV}]$ & 10.9 (fix) & $N_{\mathrm{H}}\left[\times 10^{23} \mathrm{~cm}^{-2}\right]$ & $3.2_{-0.8}^{+0.9}$ & $790 / 732$ \\
\hline & & $Z$ [solar] & $0.76_{-0.31}^{+0.63}$ & $Z$ [solar] & 0.33 (fix) & Photon index & $1.54_{-0.24}^{+0.8}$ & \\
\hline & & Redshift & $0.599_{-0.006}^{+0.004}$ & & & $\mathrm{EW}$ of $\mathrm{Fe} \mathrm{I}[\mathrm{eV}]$ & $149_{-58}^{+139}$ & \\
\hline
\end{tabular}

with that in previous work (McDonald et al. 2012). The absorption corrected luminosity of $4.7 \pm 0.7 \times 10^{45} \mathrm{ergs} \mathrm{s}^{-1}$ $(2-10 \mathrm{keV})$ is slightly higher than that in McDonald et al. (2012) but their consistency cannot be examined because they did not mention its error.

A large $N_{\mathrm{H}}$, photon index in the range of 1.5-2.0, and a strong Fe I line are common features in type 2 AGNs (Awaki et al. 1991). Together with an extremely high X-ray luminosity, the central AGN of the Phoenix cluster can be regarded as a type 2 QSO. This is the second case of a type 2 QSO in a cluster after IRAS 09104+4109 (Kleinmann et al. 1988; O'Sullivan et al. 2012).

The X-ray luminosity of $2.1_{-0.8}^{+0.7} \times 10^{46} \mathrm{ergs} \mathrm{s}^{-1} \quad(14-$
$150 \mathrm{keV}$ ) is similar to the Swift/BAT result of $1.4 \pm 0.9 \times$ $10^{46} \mathrm{ergs} \mathrm{s}^{-1}$ within errors (Cusumano et al. 2010). As is seen in Figure 3, the power-law component (AGN) is dominated over the ICM plasma in the $14-150 \mathrm{keV}$ band. Therefore the luminosity given by Cusumano et al. (2010) is surely due to the type 2 QSO, and we see no large time variability in the type 2 QSO during the Swift/BAT (2004 - 2010) and the Suzaku observation (2010). Note that the luminosity estimated from the Chandra/ACIS-I spectrum by us is $L_{\mathrm{X}}=4.0 \pm 0.5 \times 10^{45} \mathrm{ergs} \mathrm{s}^{-1}$ in $2-10 \mathrm{keV}$ with the $N_{\mathrm{H}}$ and $\Gamma$ are fixed to the best-fit parameters of Table 1, which is also consistent with the result of simultaneous fit.

Although the X-ray luminosity is exceptionally large as 


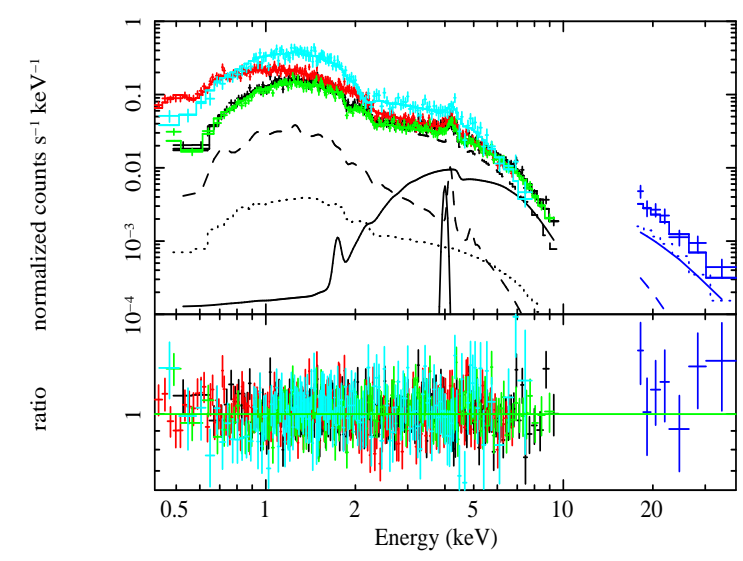

Figure 3. Suzaku/XIS \& HXD and Chandra/ACIS-I spectra simultaneously fitted with a $2 \mathrm{~T}+\mathrm{absPL}+\mathrm{Fe}$ I model expressing the cluster thermal emission and the central AGN emission. The same colors and lines are used as those in Figure 2 for the Suzaku/XIS0, XIS1. XIS3, and HXD data. The Chandra/ACIS-I data is plotted in cyan. The solid lines show an absorbed powerlaw component and a neutral iron K-line (Fe I) for XISO and HXD.

$10^{45} \operatorname{ergs~s}^{-1}(2-10 \mathrm{keV})$, the $N_{\mathrm{H}}$ and EW are on the general trend of the correlation shown in Fukazawa et al. (2011), who compiled the Suzaku results of 88 AGNs. The X-ray spectra of the type 1 and type 2 AGNs are interpreted in the unified scheme of AGNs. The differences in the observational properties of type 1 and type 2 AGNs are primarily due to observers' line of sight: face on (type 1) or edge on (type 2) to the molecular torus surrounding the nucleus. In this scheme, the X-ray spectra of type 2 AGNs consist of two components; one is penetrating through the torus, and the other is scattered at the surface of the torus. The former is called a direct component, and the latter is a reflection component. The Fe I line is mainly associated with the reflection component. Since our spectral fit in section 3 implicitly assumed that the continuum flux of the AGN is dominated by the direct component only, we try the two-component structure of the type 2 QSO with the pexmon model (Nandra et al. 2007) in XSPEC. The pexmon model represents an exponentially cut-off power-law spectrum reflected from neutral material. The direct, reflection components, and the fluorescent lines of Fe-K and Ni-K are included with a self-consistent manner. We assume the inclination angle, the cut-off energy of the power-law component, and the $\mathrm{Fe}$ abundance to be $\theta_{\mathrm{i}}=60^{\circ}, 300 \mathrm{keV}$, and 1 solar, respectively. Then, the X-ray spectra of the AGN component is reproduced with the best-fit value for the reflection fraction $(R)$ of $0.77_{-0.38}^{+0.48}$, where $R$ is defined as the ratio of the solid angle of the reflector $\Omega$ to $2 \pi$ steradian, i.e., $R=\Omega / 2 \pi$. This $R$ value and the initial assumption of viewing angle of $\theta_{\mathrm{i}}=60^{\circ}$ are consistent with the type 2 AGN picture of the torus edge-on geometry.

Ricci et al. (2011) derived $R$ values for 165 Seyfert galaxies using the hard X-ray spectra with INTEGRAL/IBIS \& ISGRI. They showed that Seyfert galaxies with $10^{23} \mathrm{~cm}^{-2}<$ $N_{\mathrm{H}}<10^{24} \mathrm{~cm}^{-2}$ (Compton thin AGNs), have $R$ of $2.2_{-1.1}^{+4.5}$ on average, which are consistent with the type 2 QSO in the Phoenix cluster within large uncertainties. Ikeda et al. (2009) performed Monte Carlo simulations of the X-ray spectra in various torus geometries. The absorption column density and the EW of the Fe I line for the type 2 QSO $\left(149_{-58}^{+139} \mathrm{eV}\right)$ in the Phoenix cluster are consistent with those from the simulation for the torus half-opening angle of $30^{\circ}$ (see the left panel of Figure 13 in Ikeda et al. 2009). Then, the expected EW of the
Fe I line is $\sim 200 \mathrm{eV}$.

The left panel of Figure 4 shows a relation of the EW of the Fe I line and the absorption column density $\left(N_{\mathrm{H}}\right)$ for our results of the Phoenix cluster and 86 Seyfert galaxies data (Fukazawa et al. 2011, but we excluded the AGN data of which no significant $N_{\mathrm{H}}$, X-ray luminosity, and the EW of Fe I were determined). The type 2 QSO in the Phoenix cluster is on the same trend of these type 1 and type 2 AGNs. In general, a higher X-ray luminosity AGN exhibits a smaller EW of the Fe I line, known as the X-ray Baldwin effect (Iwasawa \& Taniguchi 1993). The right panel of Figure 4 shows the X-ray Baldwin effect by Fukazawa et al. (2011) (the data selection is the same as the left panel of Figure 4). The type 2 QSO in the Phoenix cluster has exceptionally larger flux than any other high luminosity AGNs. Extrapolation of these data points by a linear function to the higher luminosity of $\sim 10^{46} \mathrm{ergs} \mathrm{s}^{-1}$, gives the $\mathrm{EW}$ to be no larger than a few $10 \mathrm{eV}$, far smaller than that of the type 2 QSO in the Phoenix cluster of $\mathrm{EW}=149_{-58}^{+139} \mathrm{eV}$. These indicate that the type 2 QSO has a torus of larger covering factor than those of the general trend of bright AGNs (see Figure 5 of Fukazawa et al. 2011).

\subsection{Type $2 Q S O$ - ICM interaction}

In subsection 4.3, we show that the AGN in the BCG of the Phoenix cluster is a type 2 QSO with an unobscured X-ray luminosity of $4.7 \pm 0.7 \times 10^{45} \mathrm{ergs} \mathrm{s}^{-1}(2-10 \mathrm{keV})$. The EW of the Fe I line of $\sim 150 \mathrm{eV}$ is exceptionally large for the objects with such high luminosity. Furthermore, since the type 2 fraction is smaller for higher luminosity (e.g. Ueda et al. 2003; Hasinger 2008), type 2 QSO in the Phoenix cluster is a rare case. This may be related to other extraordinary properties of this object, i.e., a very active $\mathrm{SMBH}$ in the BCG, a huge cooling rate of the ICM, and a large star formation rate in the BCG.

Assuming the bolometric correction factor of 130 (Marconi et al. 2004), the bolometric luminosity of this type 2 QSO is $L_{\text {bol.QSO }}=6.2 \pm 0.9 \times 10^{47} \mathrm{ergs} \mathrm{s}^{-1}$, which corresponds to $\sim 27 \%$ of the Eddington limit. Adopting the accretion efficiency of $\eta=0.1$, the accretion rate is estimated to be $\sim 110 M_{\odot} \mathrm{yr}^{-1}$. This is $\sim 5 \%$ of the ICM cooling rate. Other $35 \%$ of the cooled gas may be consumed by the violent star formation $\left(\sim 798 M_{\odot} \mathrm{yr}^{-1}\right.$, McDonald et al. 2013) in the BCG. We denote these two rates as $\epsilon_{\mathrm{acc}}$ and $\epsilon_{\mathrm{SFR}}$. The sum of $\epsilon_{\mathrm{acc}}$ and $\epsilon_{\mathrm{SFR}}(40 \%)$ is larger than typical cool-core clusters.

The mass of the torus can be estimated using the observed column density of $3.2 \times 10^{23} \mathrm{~cm}^{-2}$ and assuming a spherical ring with the inner radius of $0.9 \mathrm{pc}$. The inner radius of the torus is given by the $0.03 L_{43}^{0.5} \mathrm{pc}$ relation Suganuma et al. 2006, where $L_{43}$ is X-ray luminosity in unit of $10^{43} \mathrm{ergs} \mathrm{s}^{-1}$ ). The estimated torus mass is however largely dependent on the assumed outer radius, such as $3.0 \times 10^{4} M_{\odot} 1.2 \times 10^{6} M_{\odot}$ and $1.1 \times 10^{8} M_{\odot}$, and $1.1 \times 10^{10} M_{\odot}$ for the outer radius of $1 \mathrm{pc}, 10 \mathrm{pc}, 100 \mathrm{pc}$, and $1000 \mathrm{pc}$, respectively. If the accretion rate of $110 M_{\odot} \mathrm{yr}^{-1}$ has been constantly supplied by the torus, the torus mass is exhausted within $3 \times 10^{2}-1 \times 10^{8} \mathrm{yr}$, shorter than the evolution time of a SMBH, BCG, and cluster. Furthermore, if the outer radius is smaller than $1000 \mathrm{pc}$, the lifetime of this torus is shorter than that of nominal QSO lifetime of 10-20 Myr reported by Hopkins et al. (2005). Possibly, some fraction of the cooling gas would be supplied continuously to the torus. Taniguchi et al. (1997) ap- 

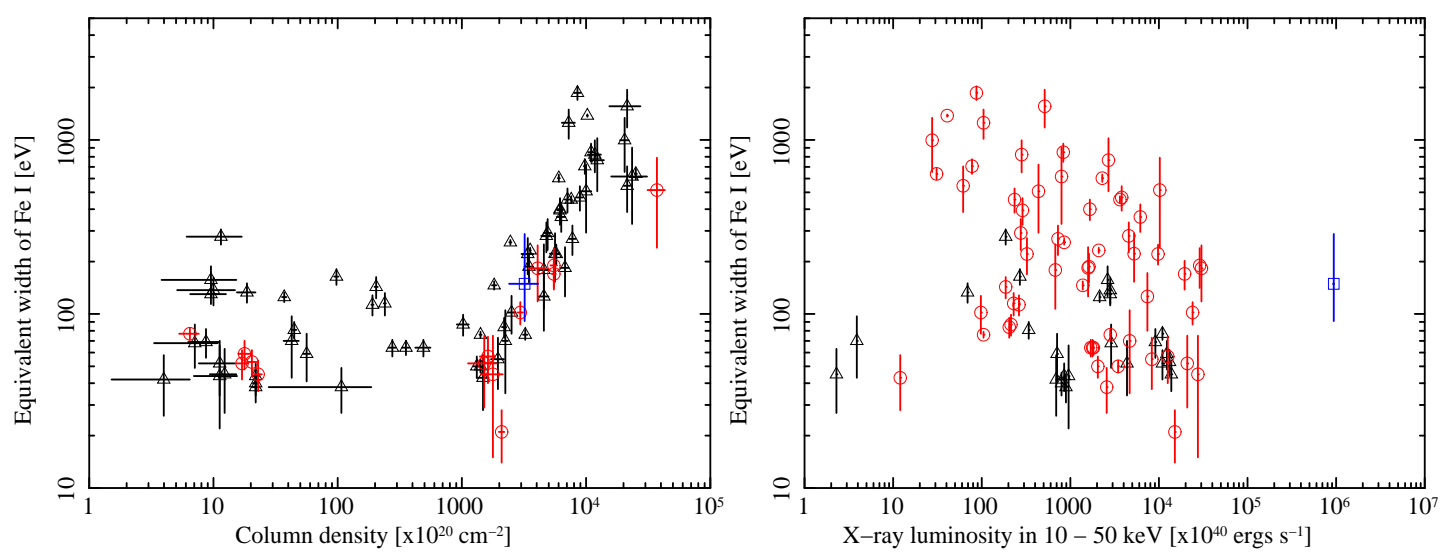

Figure 4. Left panel: relation of the $\mathrm{EW}$ of the $\mathrm{Fe} \mathrm{I}$ line and absorption column density $\left(N_{\mathrm{H}}\right)$. The black triangles are AGNs with X-ray luminosity in $10-50 \mathrm{keV}$ $\left(L_{\mathrm{X}, 10-50}\right)$ of $L_{\mathrm{X}, 10-50}<10^{44} \mathrm{ergs} \mathrm{s}^{-1}$ (Fukazawa et al. 2011, but we excluded the AGN data of which no significant $N_{\mathrm{H}}$, X-ray luminosity, and the EW of Fe I were determined), while the red circles are $L_{\mathrm{X}, 10-50}>10^{44} \mathrm{ergs} \mathrm{s}^{-1}$. The blue square shows the type 2 QSO in the Phoenix cluster (this work). Right panel: relation of the EW of the Fe I line and the X-ray luminosity in 10-50 keV. The black triangles represent the AGNs with $N_{\mathrm{H}}<10^{22} \mathrm{~cm}^{-2}$, the red circles are $N_{\mathrm{H}}$ $>10^{22} \mathrm{~cm}^{-2}$, and the blue square is the type 2 QSO in the Phoenix cluster.

plied this idea to IRAS 09104+4109 as a dust-enshrouded type 2 QSO in the center of a massive cooling-flow cluster (e.g. Fabian \& Crawford 1995; Crawford \& Vanderriest 1996; O'Sullivan et al. 2012) and estimated the mass of the torus to be $\sim 1 \times 10^{7} \quad M_{\odot}$ for a compact torus less than $10 \mathrm{pc}$, or $\sim 1 \times 10^{9} M_{\odot}$ for an extended torus of $\sim 100 \mathrm{pc}$. Fabian \& Crawford (1990) shows that a QSO in the cluster center can be fueled in a self-sustaining way through Compton cooling of the surrounding the ICM. In the type $2 \mathrm{QSO}$ of the Phoenix cluster, the cooled gas would be also supplied continuously to the torus, and would finally accrete on the SMBH.

In idealized cool-core clusters, a large cooling rate should be converted to the cooling flow, which finally should appear as significant cold gas near at the cluster center. However no clear evidence for the fate of cold gas has been observed (e.g. Makishima et al. 2001; Tamura et al. 2001; Peterson et al. 2001). This is called as the "cooling flow problem" (e.g. Fabian 1994). Some unknown mechanisms to suppress the cooling flow should be working. The most plausible explanation for the suppression of the cooling flow is heating by the AGN activity (called the "AGN feedback") (e.g. Fabian 1994; McNamara \& Nulsen 2007). Its alternative is conduction of heat from the outer part of the ICM (e.g. Fabian 2003).

As we noted, the masses responsible to the violent star formation and accretion on the SMBH is $40 \%$ of the cooling rate. The rest of $60 \%$ of the gas may be cooled and deposited in the ICM within $100 \mathrm{kpc}$ to the BCG scale. The point here is that the fraction of $40 \%$ is significantly higher than those of other nominal cool-core clusters $(\sim 10 \%$ or less, Blanton et al. 2003; McDonald et al. 2011, 2012). A question is why the cooling flow suppression, i.e., the AGN feedback, in the Phoenix cluster is less effective than the other coolcore clusters. As one possibility, McDonald et al. (2012) proposed that the Phoenix cluster is in a very rare epoch in the $\mathrm{SMBH}, \mathrm{BCG}$, and cluster evolutions, where the SMBH is powered by the cooling flow, but has not yet fully coupled with the ICM. Hence the quenching fraction of the total cooling is smaller than those in typical nearby cool-core clusters. If the AGN feedback is mainly due to jet interaction with the ICM (Perucho et al. 2011), so called the kinetic-mode (Fabian 2012), the time from the latest onset of AGN activity must be shorter than the light-crossing time of the cluster core, i.e., $\sim 0.3 \mathrm{Myrs}$. This is very short compared with typical timescale of AGN activity, and the Phoenix cluster must be in a very rare epoch.

Alternatively, the AGN feedback to the ICM can take place through radiation from the $\mathrm{QSO}$, called the radiative-mode or quasar-mode (Fabian 2012). As mentioned in Fabian (2012), this mode must be very important in the distant Universe but hard to be observed in nearby Universe. The Phoenix cluster is thus an exceptional and possibly very important case. On this point, we suggest that inefficient AGN feedback in the Phoenix cluster is related to the larger EW of the Fe I line than that predicted from the general trend of the X-ray Baldwin effect. Possible explanation of the X-ray Baldwin effect is that strong X-rays from the central AGN would reduce the mass of the torus by the X-ray evaporation (Pier \& Voit 1995; Kallman et al. 2004; Fukazawa et al. 2011), and hence reduce the EW of the Fe I line. As mentioned above, we suggest that some fractions of the massive cooling flow are supplied to the torus to compensate such an evaporation. Then, at least, a significant part of the torus is maintained (i.e. not evaporated all neutral materials yet) against an intense irradiation of bright $\mathrm{SMBH}$. This torus may shield the radiation from the SMBH to suppress the heating of the ICM further. Although we have few observational evidence of radiative-mode AGN feedback from a central AGN to ICM, the shielding effect by the torus might be an important mechanism in SMBH and BCG evolution.

\section{SUMMARY}

We have studied the X-ray spectra of the Phoenix cluster observed with the Suzaku/XIS \& HXD and the Chandra/ACIS-I, and have separately determined the ICM components and the central AGN component. We confirmed that the ICM can be approximated by a low temperature $(k T=$ $\left.2.95_{-0.48}^{+0.53} \mathrm{keV}\right)$ and high temperature $\left(k T=10.9_{-1.1}^{+1.8} \mathrm{keV}\right)$ components. The low temperature component is concentrated at the cluster core and has a high abundance of $0.76_{-0.31}^{+0.63}$ solar, while the high temperature component distributes over the cluster and has a abundance of $0.33_{-0.16}^{+0.18}$ solar. These properties of the ICM are similar to those observed in nearby coolcore clusters. The major difference is its huge cooling rate of 
$\dot{M}_{\text {total }}=2290_{-770}^{+1260} M_{\odot} \mathrm{yr}^{-1}$.

The X-ray spectrum of the central AGN in the Phoenix cluster is characterized with an strongly absorbed $\left(N_{\mathrm{H}}=3.2_{-0.8}^{+0.9} \times\right.$ $10^{23} \mathrm{~cm}^{-2}$ ) power-law continuum plus the $\mathrm{K}$-shell line from a neutral iron (Fe I). The EW of the Fe I line $\left(149_{-58}^{+139} \mathrm{eV}\right)$ and the absorption column density are typical for Compton-thin type 2 AGNs. However the EW is significantly larger than that of the general trend of the X-ray Baldwin effect, extrapolated to the luminosity as high as that of the type 2 QSO in the Phoenix cluster.

We are grateful to the anonymous referee for helpful suggestions and comments. We thank all members of the Suzaku \& Chandra operation and calibration teams. SU is supported by Japan Society for the Promotion of Science (JSPS) Research Fellowship for Young Scientist (A2411900). This work is also supported by JSPS KAKENHI Grant Number $23340071(\mathrm{KH}), 24684019(\mathrm{HN})$, and $23000004(\mathrm{HT})$.

Facilities: Suzaku (XIS, HXD) Chandra (ACIS-I).

\section{REFERENCES}

Anders, E., \& Grevesse, N. 1989, Geochim. Cosmochim. Acta, 53, 197 Angulo, R. E., Springel, V., White, S. D. M., et al. 2012, MNRAS, 425, 2722 Awaki, H., Koyama, K., Inoue, H., \& Halpern, J. P. 1991, PASJ, 43, 195 Blanton, E. L., Sarazin, C. L., \& McNamara, B. R. 2003, ApJ, 585, 227 Boldt, E. 1987, Phys. Rep., 146, 215

Crawford, C. S., \& Vanderriest, C. 1996, MNRAS, 283, 1003

Cusumano, G., La Parola, V., Segreto, A., et al. 2010, A\&A, 524, A64

De Lucia, G., \& Blaizot, J. 2007, MNRAS, 375, 2

Di Matteo, T., Allen, S. W., Fabian, A. C., Wilson, A. S., \& Young, A. J. 2003, ApJ, 582, 133

Fabian, A. C. 1994, ARA\&A, 32, 277

Fabian, A. C. 2003, in Revista Mexicana de Astronomia y Astrofisica Conference Series, Vol. 17, Revista Mexicana de Astronomia y Astrofisica Conference Series, ed. V. Avila-Reese, C. Firmani, C. S. Frenk, \& C. Allen, 303-313

-. 2012, ARA\&A, 50, 455

Fabian, A. C., \& Crawford, C. S. 1990, MNRAS, 247, 439

-. 1995, MNRAS, 274, L63

Ferrarese, L., \& Merritt, D. 2000, ApJ, 539, L9

Fukazawa, Y., Hiragi, K., Mizuno, M., et al. 2011, ApJ, 727, 19

Garmire, G. P., Bautz, M. W., Ford, P. G., Nousek, J. A., \& Ricker, Jr., G. R. 2003, in Society of Photo-Optical Instrumentation Engineers (SPIE) Conference Series, Vol. 4851, Society of Photo-Optical Instrumentation Engineers (SPIE) Conference Series, ed. J. E. Truemper \& H. D. Tananbaum, 28-44

Gebhardt, K., Bender, R., Bower, G., et al. 2000, ApJ, 539, L13

Graham, A. W., \& Scott, N. 2013, ApJ, 764, 151

Gültekin, K., Richstone, D. O., Gebhardt, K., et al. 2009, ApJ, 698, 198

Hasinger, G. 2008, A\&A, 490, 905

Hopkins, P. F., Bundy, K., Hernquist, L., \& Ellis, R. S. 2007, ApJ, 659, 976

Hopkins, P. F., Hernquist, L., Martini, P., et al. 2005, ApJ, 625, L71

Husband, K., Bremer, M. N., Stanway, E. R., et al. 2013, MNRAS, 432, 2869

Ikeda, S., Awaki, H., \& Terashima, Y. 2009, ApJ, 692, 608

Ishisaki, Y., Maeda, Y., Fujimoto, R., et al. 2007, PASJ, 59, 113

Iwasawa, K., Crawford, C. S., Fabian, A. C., \& Wilman, R. J. 2005, MNRAS, 362, L20

Iwasawa, K., Fabian, A. C., \& Ettori, S. 2001, MNRAS, 321, L15

Iwasawa, K., \& Taniguchi, Y. 1993, ApJ, 413, L15
Jiménez-Bailón, E., Santos-Lleó, M., Piconcelli, E., et al. 2007, A\&A, 461, 917

Kalberla, P. M. W., Burton, W. B., Hartmann, D., et al. 2005, A\&A, 440, 775

Kallman, T. R., Palmeri, P., Bautista, M. A., Mendoza, C., \& Krolik, J. H. 2004, ApJS, 155, 675

Kii, T., Williams, O. R., Ohashi, T., et al. 1991, ApJ, 367, 455

Kleinmann, S. G., Hamilton, D., Keel, W. C., et al. 1988, ApJ, 328, 161

Kokubun, M., Makishima, K., Takahashi, T., et al. 2007, PASJ, 59, 53

Koyama, K., Hyodo, Y., Inui, T., et al. 2007a, PASJ, 59, 245

Koyama, K., Tsunemi, H., Dotani, T., et al. 2007b, PASJ, 59, 23

Li, Y., Hernquist, L., Robertson, B., et al. 2007, ApJ, 665, 187

Makishima, K., Ezawa, H., Fukuzawa, Y., et al. 2001, PASJ, 53, 401

Marconi, A., \& Hunt, L. K. 2003, ApJ, 589, L21

Marconi, A., Risaliti, G., Gilli, R., et al. 2004, MNRAS, 351, 169

Matsushita, K. 2011, A\&A, 527, A134

McConnell, N. J., \& Ma, C.-P. 2013, ApJ, 764, 184

McConnell, N. J., Ma, C.-P., Gebhardt, K., et al. 2011, Nature, 480, 215

McConnell, N. J., Ma, C.-P., Murphy, J. D., et al. 2012, ApJ, 756, 179

McDonald, M., Benson, B., Veilleux, S., Bautz, M. W., \& Reichardt, C. L. 2013, ApJ, 765, L37

McDonald, M., Veilleux, S., Rupke, D. S. N., Mushotzky, R., \& Reynolds,

C. 2011, ApJ, 734, 95

McDonald, M., Bayliss, M., Benson, B. A., et al. 2012, Nature, 488, 349

McLure, R. J., \& Dunlop, J. S. 2002, MNRAS, 331, 795

McNamara, B. R., \& Nulsen, P. E. J. 2007, ARA\&A, 45, 117

Mitsuda, K., Bautz, M., Inoue, H., et al. 2007, PASJ, 59, 1

Morrison, R., \& McCammon, D. 1983, ApJ, 270, 119

Mushotzky, R. F., Done, C., \& Pounds, K. A. 1993, ARA\&A, 31, 717

Nandra, K., O’Neill, P. M., George, I. M., \& Reeves, J. N. 2007, MNRAS, 382, 194

O'Sullivan, E. Giacintucci, S., Babul, A et al. 2012, MNRAS, 424, 2971

Ota, N. 2012, Research in Astronomy and Astrophysics, 12, 973

Ota, N., Fukazawa, Y., Fabian, A. C., et al. 2007, PASJ, 59, 351

Ozawa, M., Uchiyama, H., Matsumoto, H., et al. 2009, PASJ, 61, 1

Perucho, M., Quilis, V., \& Martí, J.-M. 2011, ApJ, 743, 42

Peterson, J. R., Paerels, F. B. S., Kaastra, J. S., et al. 2001, A\&A, 365, L104

Pier, E. A., \& Voit, G. M. 1995, ApJ, 450, 628

Ricci, C., Walter, R., Courvoisier, T. J.-L., \& Paltani, S. 2011, A\&A, 532, A102

Richards, G. T., Strauss, M. A., Fan, X., et al. 2006, AJ, 131, 2766

Russell, H. R., Fabian, A. C., Sanders, J. S., et al. 2010, MNRAS, 402, 1561

Russell, H. R., Fabian, A. C., Taylor, G. B., et al. 2012, MNRAS, 422, 590

Sadoun, R., \& Colin, J. 2012, MNRAS, 426, L51

Salpeter, E. E. 1964, ApJ, 140, 796

Salviander, S., \& Shields, G. A. 2013, ApJ, 764, 80

Siemiginowska, A., Burke, D. J., Aldcroft, T. L., et al. 2010, ApJ, 722, 102

Skrutskie, M. F., Cutri, R. M., Stiening, R., et al. 2006, AJ, 131, 1163

Smith, R. K., Brickhouse, N. S., Liedahl, D. A., \& Raymond, J. C. 2001, ApJ, 556, L91

Springel, V., Frenk, C. S., \& White, S. D. M. 2006, Nature, 440, 1137

Springel, V., White, S. D. M., Jenkins, A., et al. 2005, Nature, 435, 629

Suganuma, M., Yoshii, Y., Kobayashi, Y., et al. 2006, ApJ, 639, 46

Takahashi, H., Hayashida, K., \& Anabuki, N. 2010, PASJ, 62, 1483

Takahashi, T., Abe, K., Endo, M., et al. 2007, PASJ, 59, 35

Tamura, T., Hayashida, K., Ueda, S., \& Nagai, M. 2011, PASJ, 63, 1009

Tamura, T., Kaastra, J. S., Peterson, J. R., et al. 2001, A\&A, 365, L87

Taniguchi, Y., Sato, Y., Kawara, K., Murayama, T., \& Mouri, H. 1997, A\&A, 318, L1

Tawa, N., Hayashida, K., Nagai, M., et al. 2008, PASJ, 60, 11

Tremaine, S., Gebhardt, K., Bender, R., et al. 2002, ApJ, 574, 740

Ueda, S., Hayashida, K., Nakajima, H., \& Tsunemi, H. 2013,

Astronomische Nachrichten, 334, 426

Ueda, Y., Akiyama, M., Ohta, K., \& Miyaji, T. 2003, ApJ, 598, 886

Véron-Cetty, M.-P., \& Véron, P. 2001, A\&A, 374, 92

Vestergaard, M., Fan, X., Tremonti, C. A., Osmer, P. S., \& Richards, G. T. 2008, ApJ, 674, L1

Vignali, C., Piconcelli, E., Lanzuisi, G., et al. 2011, MNRAS, 416, 2068

Voges, W., Aschenbach, B., Boller, T., et al. 1999, A\&A, 349, 389

Volonteri, M., \& Ciotti, L. 2013, ApJ, 768, 29

White, D. A., Jones, C., \& Forman, W. 1997, MNRAS, 292, 419

Williamson, R., Benson, B. A., High, F. W., et al. 2011, ApJ, 738, 139

Yamashita, A., Matsumoto, C., Ishida, M., et al. 1997, ApJ, 486, 763 